\title{
Estudio para la instalación de una fábrica de snacks a partir de la fritura de yacón
}

\author{
Gabriela Estefanía Doig Castillo \\ Universidad de Lima. Lima, Perú \\ Correo electrónico: gabriela.doig@gmail.com
}

Recibido: 21/4/2012 / Aprobado: 15/6/2012

\begin{abstract}
RESUMEN: El yacón es un tubérculo que tiene como característica principal su alta cantidad de fructooligosacáridos o azúcares que no son metabolizados por el cuerpo humano. Se considera, entre otras bondades, su intrínseca versatilidad, que permite su industrialización para fabricar snacks (hojuelas fritas de yacón). Este estudio presenta la definición y cuantificación de la demanda, la tecnología necesaria para desarrollar el producto y una evaluación económico-financiera a fin de justificar la inversión privada rentable. Incluye, además, alternativas de desarrollo social en las zonas de influencia directa e indirecta del proyecto.
\end{abstract}

Palabras clave: Yacón / snacks / hojuelas fritas

\section{Study for the installation of a production plant of snacks made by fried yacon crisps}

\begin{abstract}
Yacon is a root, one of its main characterstic is that it contains a high proportion of fructooligosaccharides, a type of sugar which is not metabolized by the human body. This and other beneficial properties, along with yacon's intrinsic versatility which permits its diverse industrialised elaboration, are considered in the development of the present study: fabrication of snacks (fried yacon crisps). This study approaches the subject by presenting the definition and quantification of the demand, the type of technology required for the manufacturing and an economic-financial evaluation, in order to justify profitable private investment; including alternatives for social development in the direct and indirect project influence areas.
\end{abstract}

Keywords: Yacon / snacks / fried crisps 


\section{ANTECEDENTES Y MARCO TEÓRICO}

El yacón (Smallanthus sonchifolius) es un tubérculo comestible originario de las zonas andinas del norte y del centro del país. Su característica más destacable es la de poseer un tipo de azúcar no digerible por el ser humano: los fructooligosacáridos (FOS). Tal tipo de azúcar representa una opción interesante para personas que padecen diabetes u obesidad; ya que el tubérculo tiene un sabor dulce que no es peligroso para los niveles de azúcar en la sangre ni para el incremento de grasas. Entre otras propiedades, se relaciona a los FOS con un mejor funcionamiento gastrointestinal, mejor asimilación del calcio y ayuda en la reducción de los niveles de triglicéridos y colesterol.

\section{USOS Y PROPIEDADES}

Usualmente, el yacón se consume crudo, como acompañante de las ensaladas y frutas. Se podría decir que es más parecido a una fruta por las propiedades organolépticas que posee. Como producto elaborado se puede encontrar en forma de jugo, después de haber sido rallado y exprimido; en jarabes de yacón, extrayéndole y concentrando el azúcar; y como hojuelas deshidratadas. Sus hojas pueden ser utilizadas como forraje para ganado y también como infusión en bolsitas filtrantes.

En la tabla 1 puede apreciarse el contenido de calorías del yacón comparado con otros alimentos:

\section{Tabla 1}

Contenido calórico del yacón

\begin{tabular}{lc}
\hline Alimento & $\begin{array}{c}\text { Contenido calórico } \\
\text { (Kcal/100 g) }\end{array}$ \\
\hline Yacón & 17 \\
Piña & 40 \\
Manzana & 60 \\
Papa & 120 \\
Pan & 250 \\
Chocolate & 500
\end{tabular}

Fuente: Seminario, Valderrama \& Manrique (2003). 


\section{ESTUDIO DE MERCADO}

\subsection{Definición del producto}

El producto propuesto para esta investigación son los snacks fritos de yacón para el consumo masivo, enfocados principalmente en el mercado nutricional, pero no necesariamente restringidos a este nicho. Se busca resaltar las bondades que presenta el yacón como materia prima, para reducir la connotación poco saludable que conlleva la palabra snack.

La presentación consistirá en una bolsa de polipropileno biorientado, de colores claros y llamativos, que contendrá una cantidad de hojuelas fritas de yacón en atmósferas inertes. ${ }^{1}$ La presentación clásica tendrá unos 50 gramos de contenido e ingresará a competir en el mercado nacional de snacks (específicamente en Lima metropolitana) y su posterior ingreso a provincias.

\subsection{Situación relativa en el mercado}

El mercado de snacks en el ámbito nacional es muy amplio y diverso; existe una gran variedad de productos altamente posicionados, tanto dulces como salados. Pero este escenario no debe ser evaluado como una desventaja, pues se puede aprovechar el trabajo de sensibilización que los competidores iniciaron en su momento con la diversificación de sus productos, fabricando snacks elaborados con materias primas no convencionales, como el camote, el plátano, las habas, etcétera. El eventual ingreso de snacks de yacón no representará un cambio tan radical en las tendencias de lanzamiento de nuevos productos.

Los snacks o los "bocaditos" se pueden consumir solos o como acompañamiento de refrescos o bebidas alcohólicas en las reuniones sociales.

\subsection{Análisis de la demanda}

Dado que el producto propuesto aún no existe en el mercado, se tomó como punto de partida la demanda general de snacks en el Perú. En cuanto a las importaciones, se obtuvieron los datos del portal de la Superintendencia Nacional de Aduanas y de Administración Tributaria (Sunat), bajo las respectivas partidas arancelarias. Los datos estadísticos se muestran en el gráfico 1 :

1 Si las hojuelas quedan expuestas al aire, es posible que, por oxidación, se ablanden y rancien. Una atmósfera inerte con $\mathrm{N}_{2} \circ \mathrm{CO}_{2}$ evitaría este problema. 
Gráfico 1

Evolución de las importaciones según país de procedencia (toneladas)

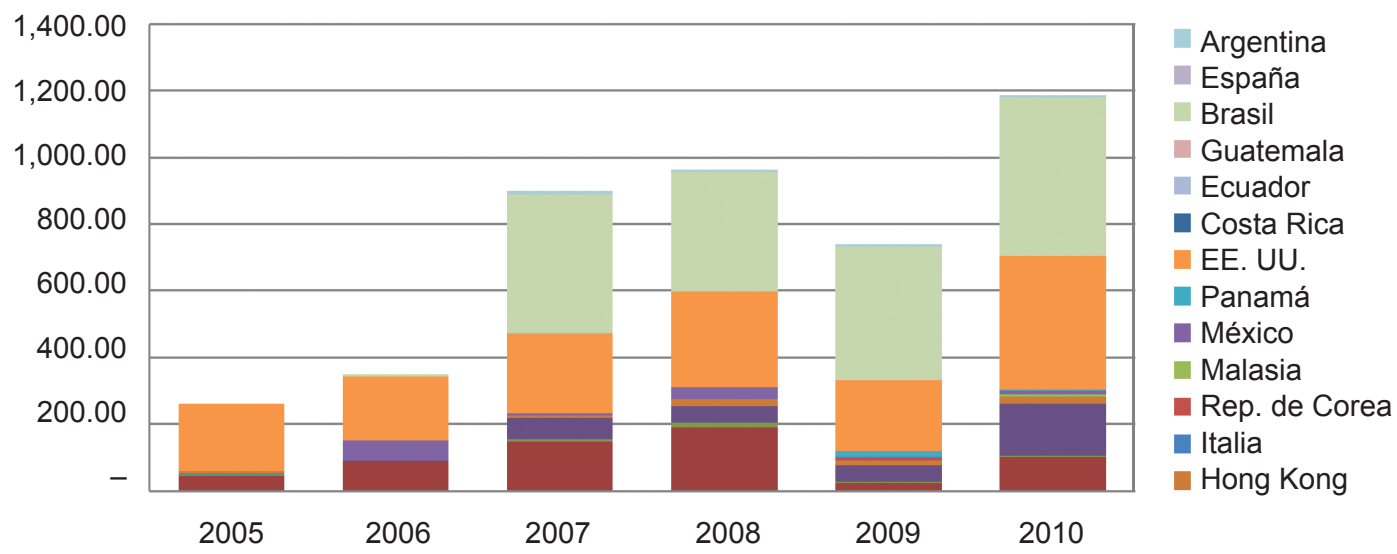

Fuente: Sunat (2011).

Elaboración propia.

En el gráfico se observa que la tendencia se mantuvo generalmente estable con una disminución en el año 2009 y un considerable repunte en el 2010.

En cuanto a la producción nacional, y siguiendo las cifras del Ministerio de la Producción (Produce), se obtuvo la siguiente información, que se muestra en el gráfico 2.

\section{Gráfico 2}

Producción de snacks 2005-2010 (toneladas)

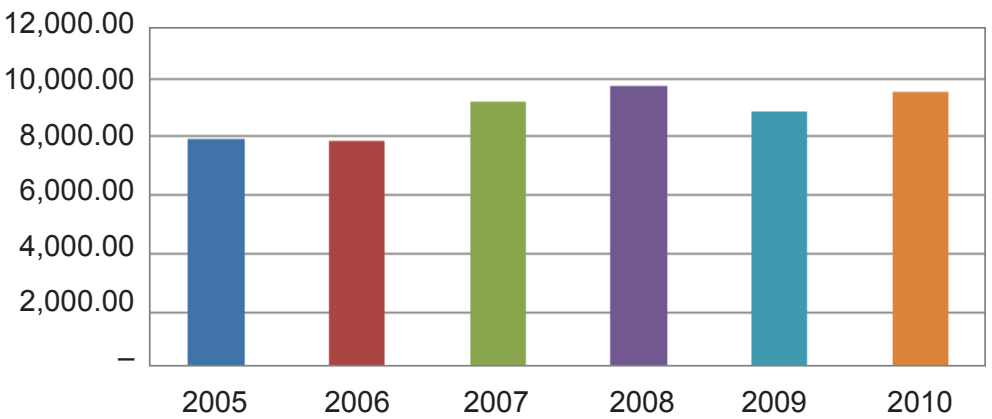

Fuente: Produce (2011).

Elaboración propia. 
En el gráfico se puede visualizar que el crecimiento porcentual total en el periodo analizado es del $20 \%$, brindando referencia respecto al mercado y su aparente expansión.

\subsection{Demanda potencial}

Para tener un marco referencial, en la tabla siguiente se presenta el consumo per cápita en el Perú, comparado con el de otros países de la región latinoamericana y los Estados Unidos.

Tabla 2

Consumo per cápita de snacks (g-persona/año)

\begin{tabular}{lr}
\hline \multicolumn{1}{c}{ País } & CPC \\
\hline Perú & 350 \\
Chile & 900 \\
México & 4000 \\
Estados Unidos & 8000 \\
\hline
\end{tabular}

Fuente: Armas, Granados \& Puente (2004).

Elaboración propia.

Si bien el consumo viene en aumento, se puede afirmar que el mercado peruano es aún pequeño comparado con el de los demás países. Esto representa una ventaja, ya que este no está tan saturado como los otros. Como punto de referencia para el cálculo de la demanda potencial, se toma el caso chileno:

Tabla 3

Demanda potencial (toneladas)

\begin{tabular}{ccc}
\hline $\begin{array}{c}\text { Población urbana } \\
\text { Perú* }\end{array}$ & $\begin{array}{c}\text { Consumo per cápita } \\
\text { g/persona }\end{array}$ & $\begin{array}{c}\text { Demanda potencial }= \\
\text { población } \mathbf{x} \text { CPC }\end{array}$ \\
\hline 21.986 .611 & 350 (Perú) & 7695 \\
21.986 .611 & 900 (Chile) & 19.788 \\
\hline
\end{tabular}

*Proyectada al 2010.

Elaboración propia. 
Se puede afirmar que la demanda potencial en el sector de snacks podría llegar a las 19.788 toneladas anuales, según lo mostrado en la tabla 3 ; sin embargo, para tener cifras más exactas, se realizará el cálculo de la demanda interna aparente.

\subsection{Demanda interna aparente}

El cálculo de la demanda interna aparente se realizará considerando los datos de importación y exportación en el Perú obtenidos en el portal de la Aduana.

Tabla 4

Demanda aparente (toneladas)

\begin{tabular}{rrrrrrrr}
\hline & $\begin{array}{c}\text { Producción } \\
\text { de snacks }\end{array}$ & & Importaciones & & Exportaciones & $\begin{array}{c}\text { Demanda } \\
\text { aparente }\end{array}$ \\
\hline 2005 & $7.936,57$ & + & 259,96 & - & 64,98 & $=$ & $8.131,55$ \\
2006 & $7.882,07$ & + & 341,61 & - & 64,11 & $=$ & $8.159,57$ \\
2007 & $9.204,54$ & + & 895,91 & - & 126,69 & $=$ & $9.973,76$ \\
2008 & $9.757,54$ & + & 964,90 & - & 253,78 & $=$ & $10.468,66$ \\
2009 & $8.860,53$ & + & 734,31 & - & 324,06 & $=$ & $9.270,78$ \\
2010 & $9.526,44$ & + & $1.181,48$ & - & 268,07 & $=$ & $10.439,85$ \\
\hline
\end{tabular}

Fuente: Sunat.

Elaboración propia.

\subsubsection{Proyección de la demanda}

Tomando los datos hallados de demanda aparente, la proyección de esta para los próximos diez años será la siguiente, aplicando una regresión potencial. 
Tabla 5

Proyección de la demanda a 10 años (ámbito nacional)

\begin{tabular}{cc}
\hline Año & $\begin{array}{c}\text { Demanda aparente } \\
\text { toneladas }\end{array}$ \\
\hline 2005 & $8.131,55$ \\
2006 & $8.159,57$ \\
2007 & $9.973,76$ \\
2008 & $10.468,66$ \\
2009 & $9.270,78$ \\
2010 & $10.439,85$ \\
2011 & $11.066,93$ \\
2012 & $11.610,35$ \\
2013 & $12.180,16$ \\
2014 & $12.777,63$ \\
2015 & $13.404,10$ \\
2016 & $14.060,94$ \\
2017 & $14.749,62$ \\
2018 & $15.471,67$ \\
2019 & $16.228,67$ \\
2020 & $17.022,32$ \\
2021 & $17.854,35$ \\
\hline
\end{tabular}

Elaboración propia.

\subsection{Análisis de la oferta}

El mercado de snacks en el Perú es relativamente pequeño comparado con el de otros países, pero se encuentra en expansión y representa una oportunidad para poder diversificar la actual oferta. Para ello, se requiere desarrollar dos ideas principales:

- El abastecimiento de materia prima e insumos se garantizará cuando se logre administrar agrupaciones de productores en sectores estratégicos del país.

- Se debe aprovechar el crecimiento de la demanda por productos naturales nativos y sanos como tendencia del mercado. 


\subsubsection{Proyección de la oferta}

Debido a que no existe información oficial disponible, se presume que la oferta se encuentra representada por la producción nacional; de esa manera, se proyecta la oferta a través de una regresión potencial para los próximos diez años:

Tabla 6

Proyección de la oferta a 10 años

\begin{tabular}{cc}
\hline Año & Oferta (toneladas) \\
\hline 2005 & $7.936,57$ \\
2006 & $7.882,07$ \\
2007 & $9.204,54$ \\
2008 & $9.757,54$ \\
2009 & $8.860,53$ \\
2010 & $9.526,44$ \\
2011 & $10.078,60$ \\
2012 & $10.465,94$ \\
2013 & $10.867,96$ \\
2014 & $11.285,22$ \\
2015 & $11.718,27$ \\
2016 & $12.167,71$ \\
2017 & $12.634,16$ \\
2018 & $13.118,24$ \\
2019 & $13.620,62$ \\
2020 & $14.141,98$ \\
2021 & $14.683,01$ \\
\hline
\end{tabular}

Elaboración propia.

\subsubsection{Determinación de la demanda para el proyecto}

Se realizó la mayor cantidad de encuestas ${ }^{2}$ tomando como referencia el tamaño de la muestra previamente calculado. Para cuantificar la

2 Al no poderse realizar una encuesta con una muestra representativa ( $\mathrm{n}>=400)$ por limitaciones de tiempo y dinero, se buscó realizar el máximo de encuestas posibles, logrando una muestra de $\mathrm{n}=120$. Los resultados son referenciales y de ninguna manera representativos. 
demanda, se consideró el porcentaje de intención de compra afirmativa que arrojó la encuesta, como se observa en la siguiente tabla:

Tabla 7

Intención de compra

\begin{tabular}{lcc}
\hline Decisión & Cantidad & Porcentaje \\
\hline Sí & $\mathbf{9 4}$ & $\mathbf{7 8 , 3 3}$ \\
No & 9 & 7,50 \\
Tal vez & 17 & 14,17 \\
Total & 120 & 100,00 \\
\hline
\end{tabular}

Fuente: Doig (2011).

Elaboración propia.

Luego se multiplicó por el porcentaje de productos susceptibles de ser sustituidos (véase la tabla 8):

Tabla 8

Preferencia de snacks

\begin{tabular}{lcc}
\hline Snack & Cantidad “Favorito" & Porcentaje \\
\hline Papas Lay & 24 & 22,43 \\
Maní & $\mathbf{1 5}$ & $\mathbf{1 4 , 0 2}$ \\
Chifles & $\mathbf{1 4}$ & $\mathbf{1 3 , 0 8}$ \\
Camote & $\mathbf{1 0}$ & $\mathbf{9 , 3 5}$ \\
Cuates & 8 & 7,48 \\
Cheese Triz & 8 & 7,48 \\
Torteez & 7 & 6,54 \\
Piqueo Snax & 7 & 6,54 \\
Doritos & 6 & 5,61 \\
Chizitos & 5 & 4,67 \\
Karinpops & 2 & 1,87 \\
Otros & $\mathbf{1}$ & $\mathbf{0 , 9 3}$ \\
Total & 107 & 100,00 \\
\hline
\end{tabular}

Elaboración propia. 
Demanda del proyecto $=(\%$ de aceptación $) \times(\%$ sustituible $)$

Demanda del proyecto $=78,33 \% \times(14,02 \%+13,08 \%+9,35 \%+0,93 \%)$

Demanda del proyecto $=29,28 \% \times$ Oferta actual

Demanda del proyecto $=29,28 \% \times 9,526.44$ toneladas $^{3}$

Demanda del proyecto $=2789,34=2790$ toneladas anuales

Sin embargo, se asume una posición más conservadora tomando aproximadamente un tercio del porcentaje obtenido, por las siguientes razones:

- No se cuenta con información estadísticamente válida. La encuesta realizada no es representativa debido al reducido tamaño de muestra obtenida y los resultados son referenciales.

- En el porcentaje sustituible de demanda se incluyen tanto snacks dulces como salados y picantes (maní y chifles). En este caso, no se puede asumir que el total de la aceptación obtenida es discriminado por algún sabor en particular; por ello, no se toma el porcentaje completo.

- Se consideran como productos sustituibles los snacks elaborados con maní, chifles, camote, habas, cancha/maíz, etcétera, ya que se apunta a sustituir snacks con productos no tradicionales, entrando a competir directamente con ese tipo de productos. Los snacks hechos con papa, queso y tortillas de maíz se encuentran mucho mejor posicionados en el mercado.

Por lo mencionado, la demanda máxima del proyecto se estima en:

Demanda del proyecto $=10 \% \times 9,526.44$ toneladas $=953$ toneladas anuales.

\subsubsection{Análisis de precios}

Los precios se han mantenido muy estables durante los últimos años para cada presentación. La de 38 a 50 gramos (dependiendo del productor) se ha mantenido a un sol, precio bastante accesible para el público en general.

$3 \quad$ Al año 2008. 


\subsubsection{Programa de ventas para diez años}

Para poder proyectar el programa de ventas, se tomará como base la variación porcentual del PBI nacional, calculando que tendrá un impacto directo y proporcional sobre las ventas. Se consideraron los últimos tres años como referencia (sin incluir el año 2008) y se obtuvo un promedio simple de la variación $(7,8 \%)$. Con esto se preparó el programa de ventas para los siguientes diez años (tabla 9).

Tabla 9

Programa de ventas para los próximos 10 años

\begin{tabular}{lcccccccccc}
\hline (t/año) & $\mathbf{2 0 1 1}$ & $\mathbf{2 0 1 2}$ & $\mathbf{2 0 1 3}$ & $\mathbf{2 0 1 4}$ & $\mathbf{2 0 1 5}$ & $\mathbf{2 0 1 6}$ & $\mathbf{2 0 1 7}$ & $\mathbf{2 0 1 8}$ & $\mathbf{2 0 1 9}$ & $\mathbf{2 0 2 0}$ \\
\hline Demanda & 10.301 & 10.661 & 11.034 & 11.419 & 11.817 & 12.229 & 12.655 & 13.096 & 13.552 & 14.024 \\
Plan de ventas & 1.030 & 1.110 & 1.196 & 1.289 & 1.389 & 1.497 & 1.614 & 1.739 & 1.874 & 2.020 \\
Participación & 10 & 10 & 11 & 11 & 12 & 12 & 13 & 13 & 14 & 14 \\
\hline
\end{tabular}

Elaboración propia.

\section{DISPONIBILIDAD DE INSUMOS}

\subsection{Cultivos}

A pesar de haber sido descubierto y cultivado en el país desde épocas prehispánicas, de acuerdo con Seminario, Valderrama y Manrique (2003), se ha comprobado que en las tres décadas pasadas existían menos de 100 hectáreas sembradas a nivel nacional. Los mismos autores indican que el rendimiento por hectárea —en el caso peruano- está, en promedio, entre las 30 y 50 toneladas. En otros países ${ }^{4}$ se sabe que este fluctúa entre 10 y 100 toneladas. Cabe mencionar que el rendimiento se ve afectado por la densidad de siembra: a mayor densidad, mayor rendimiento pero con raíces de menor tamaño. A menor densidad sucede lo contrario: menor rendimiento pero raíces de mayor tamaño.

Esta información ha sido contrastada con los datos del Ministerio de Agricultura. En la tabla siguiente se muestra información oficial donde se observa que el rendimiento es mucho menor al indicado por Seminario et al. (2003).

4 Según pruebas documentadas en Brasil, Ecuador, Japón, Corea y Perú (Seminario, Valderrama \& Manrique, 2003). 
Tabla 10

Cultivo de yacón a nivel nacional

\begin{tabular}{lccccccccc}
\hline Total & $\mathbf{2 0 0 1}$ & $\mathbf{2 0 0 2}$ & $\mathbf{2 0 0 3}$ & $\mathbf{2 0 0 4}$ & $\mathbf{2 0 0 5}$ & $\mathbf{2 0 0 6}$ & $\mathbf{2 0 0 7}$ & $\mathbf{2 0 0 8}$ & $\mathbf{2 0 0 9}$ \\
\hline Siembras (ha) $\Sigma$ & 62 & 229 & 154 & 260 & 207 & 420 & 643 & 587 & \\
Cosechas (ha) $\Sigma$ & & 48 & 229 & 153 & 288 & 203 & 396 & 663 & 326 \\
Producción (t) $\Sigma$ & & 444 & 4.184 & 2.357 & 4.789 & 4.823 & 6.770 & 9.558 & 4.443 \\
Rendimiento (t/ha) $\dot{X}$ & & 9,3 & 14,5 & 14,6 & 13,8 & 24,1 & 10,8 & 7,1 & 8,7 \\
\hline
\end{tabular}

Fuente: Ministerio de Agricultura.

Elaboración propia.

La superficie sembrada ha aumentado con el paso de los años, debido a la creciente demanda del yacón; sin embargo, el rendimiento no ha sido el esperado. Aparentemente, las cosechas son buenas pero existen muchas pérdidas.

\subsection{Potencialidad del recurso en la zona de influencia del proyecto}

Los principales beneficiarios con el desarrollo del proyecto serían los productores de la materia prima; por eso, uno de los objetivos implícitos es llevar desarrollo a los comuneros, que en su mayoría conforman un gran porcentaje de la población en situación de pobreza en el país. La articulación con el sector industrial brindará empleo tanto directo como indirecto en los diversos puntos de la cadena de suministro que la empresa integre y mejorará el nivel de vida de las personas involucradas como parte de su desarrollo integral.

Según Seminario et al. (2003), los principales lugares o nichos de producción del yacón en el país se encuentran en Amazonas, Apurímac, Arequipa, Ayacucho, Cajamarca, Cerro de Pasco, Cusco, Huánuco, La Libertad, Lambayeque, Lima y Puno, siendo Cajamarca uno de los más importantes. Es allí donde se han registrado productividades de hasta 95 toneladas por hectárea (Seminario, 1995). Sin embargo, y según información oficial, el promedio de rendimiento país en los últimos 8 años fue de 12.86 toneladas por hectárea. Esto es sumamente inferior a lo registrado en el estudio de Seminario (1995). En la siguiente tabla se presentan los principales departamentos productores. 
Tabla 11

Cultivo de yacón por departamento (toneladas)

\begin{tabular}{lcccccccc}
\hline Año & Amazonas & Cajamarca & Cusco & La Libertad & Lima & Pasco & Puno & Total \\
\hline 2005 & 2.480 & 15 & 1.200 & 84 & 12 & SD & 998 & 4.789 \\
2006 & 2.691 & SD & 2.132 & SD & SD & SD & SD & 4.823 \\
2007 & 3.544 & 8 & 1.925 & SD & SD & 324 & 969 & 6.770 \\
2008 & 8.040 & SD & 140 & SD & SD & 249 & 1.129 & 9.558 \\
2009 & 2.550 & SD & 648 & SD & SD & 108 & 1.137 & 4.443 \\
Total & 22.831 & 59 & 7.609 & 84 & 12 & 681 & 6.093 & - \\
\hline
\end{tabular}

Fuente: Ministerio de Agricultura, data proporcionada por vía electrónica (correo electrónico, 7 de agosto del 2009).

Elaboración propia.

Se tomará como rendimiento promedio por hectárea el valor de 13 toneladas, ${ }^{5}$ por tener el presente estudio un carácter conservador.

\subsection{Participación del proyecto dentro de la disponibilidad de insumos}

Se buscará la sinergia en la cadena productiva: los proveedores garantizarán una materia prima de primera calidad y, de acuerdo con las especificaciones que se le impongan, a cambio de esto, la empresa garantizará la compra constante a precios competitivos. Al mismo tiempo, se presenta la alternativa de funcionar como avales frente a entes financieros para que de esta manera las comunidades puedan acceder a préstamos y financiamiento que permitan mejorar la producción, así como invertir en investigación y desarrollo del cultivo.

Para determinar la relación insumo/producto se tomarán los siguientes datos y consideraciones:

- La humedad promedio del yacón fluctúa entre $75 \%$ y $85 \%$ en peso, porcentajes similares a los de la papa. Este tubérculo presenta un rendimiento de aproximadamente 2,8 a $1,{ }^{6}$ es decir, por cada 2,8 kilogramos de papa fresca se obtiene 1 kilogramo de papa procesada; esto representa aproximadamente un $36 \%$ de aprovechamiento. Para propósitos prácticos, y al presentar el yacón mayor humedad que la papa, se tomará la relación de 3 a 1.

5 De acuerdo con Seminario et al. (2003), el rendimiento promedio de yacón en Cajamarca fluctúa entre 40 y 50 toneladas por hectárea.

6 Valor obtenido del Portal Agrario Ancash (2008). 
- Se tomará como valor inicial el dato de 586 hectáreas sembradas a nivel nacional al 2008, según la información obtenida del Ministerio de Agricultura. El crecimiento anual se calculó mediante una regresión lineal utilizando datos históricos.

Tabla 12

Relación insumo/producto

\begin{tabular}{ccccc}
\hline Año & $\begin{array}{c}\text { Plan de } \\
\text { ventas }(\mathbf{t})\end{array}$ & $\begin{array}{c}\text { Requerimiento } \\
\text { MP - yacón }(\mathbf{t})\end{array}$ & $\begin{array}{c}\text { Producción } \\
\text { local }(\mathbf{t})\end{array}$ & $\begin{array}{c}\text { Porcentaje } \\
\text { de participación }\end{array}$ \\
\hline 2011 & $1.030,13$ & $3.090,40$ & $11.721,30$ & 26 \\
2012 & $1.110,15$ & $3.330,46$ & $12.868,19$ & 26 \\
2013 & $1.196,39$ & $3.589,16$ & $14.015,08$ & 26 \\
2014 & $1.289,32$ & $3.867,96$ & $15.161,97$ & 26 \\
2015 & $1.389,47$ & $4.168,41$ & $16.308,86$ & 26 \\
2016 & $1.497,40$ & $4.492,21$ & $17.455,75$ & 26 \\
2017 & $1.613,72$ & $4.841,15$ & $18.602,64$ & 26 \\
2018 & $1.739,07$ & $5.217,20$ & $19.749,52$ & 26 \\
2019 & $1.874,15$ & $5.622,46$ & $20.896,41$ & 27 \\
2020 & $2.019,74$ & $6.059,21$ & $22.043,30$ & 27 \\
\hline
\end{tabular}

Elaboración propia.

\subsection{Costos de la materia prima e insumos}

De acuerdo con la información publicada en el portal del Ministerio de Agricultura, el precio del kilogramo de yacón en el Mercado Mayorista número 2 (Mercado de Frutas) se encuentra en S/.1,50 en promedio, habiéndose mantenido estable en los últimos meses (Ministerio de Agricultura, datos a julio del 2009). En cuanto al precio en los mercados minoristas, se pudo comprobar que bordea los S/.3,60 el kilogramo, mientras que en la chacra este ha tenido la evolución que se observa en tabla 13: 
Tabla 13

Evolución del precio promedio en los últimos años

\begin{tabular}{cc}
\hline Total & $\begin{array}{c}\text { Precio chacra } \\
\text { (S/. x kg) (promedio) }\end{array}$ \\
\hline 2005 & 0,48 \\
2006 & 0,59 \\
2007 & 0,34 \\
2008 & 0,23 \\
2009 & 0,32 \\
\hline
\end{tabular}

Elaboración propia.

\section{INGENIERÍA DEL PROYECTO}

\subsection{Características del producto}

El producto final consistirá en 50 gramos aproximadamente de hojuelas fritas de yacón contenidas en una bolsa de polipropileno biorientado, de unos 13 centímetros de ancho por 20 de largo. Entre los principales insumos se pueden destacar:

- Rodajas de yacón fresco, como materia prima principal.

- Aceite de palma.

- Bolsas de polipropileno biorientado (plástico flexible, que sean resistentes y que permitan bloquear el oxígeno y la humedad para garantizar la calidad y maximizar la vida útil del producto.

El producto debe conservar la frescura, las hojuelas deben estar enteras; no quebradas ni frías; es decir, deberán mantener su consistencia crocante. A continuación se detallan las principales características del producto:

Tabla 14

Principales características del producto terminado

\begin{tabular}{ll}
\hline Nombre & Snacks fritos de yacón \\
\hline Descripción & $\begin{array}{l}\text { Rodajas de yacón fritas (hojuelas), } \\
\text { preparadas para consumo directo }\end{array}$ \\
\hline Composición & Yacón, aceite de palma \\
\hline \multicolumn{2}{c}{ (continúa) }
\end{tabular}


(continuación)

\begin{tabular}{ll}
\hline $\begin{array}{l}\text { Características } \\
\text { organolépticas }\end{array}$ & $\begin{array}{l}\text { Sabor dulce, olor casi impercep- } \\
\text { tible, textura crocante, color amarillo } \\
\text { oscuro }\end{array}$ \\
\hline Presentación & $\begin{array}{l}\text { Bolsa de plástico flexible con } 50 \mathrm{~g} \text { de } \\
\text { contenido }\end{array}$ \\
\hline Vida útil & 4 meses desde el sellado de la bolsa
\end{tabular}

Elaboración propia.

\subsection{Tecnologías existentes y procesos de producción}

Al tratarse de un proceso bastante simple y conocido, la tecnología no representa una mayor limitación al momento de concebir el proceso de producción. Este proceso, en términos muy generales, sería el siguiente:

- Recepción, selección y limpieza.- Se realizará una inspección de los lotes entrantes tomando muestras para poder mantener un nivel de calidad aceptable a lo largo de todo el proceso de producción. Las dimensiones ideales del yacón son:

- Largo: No menor de 20 centímetros.

- Diámetro: No menor de 7 centímetros.

La materia prima ingresaría al proceso a través de una tolva de alimentación, pasando inmediatamente a una faja transportadora donde se realizará la selección inicial, retirando las impurezas.

Luego, pasará a la sección de limpieza conformada por una máquina lavadora en túnel que consiste en un sistema de rodillos para el traslado y una cámara de rociado de agua a alta presión (35 bar). Los rodillos también permiten que las raíces giren y, por tanto, que el lavado sea más eficiente. Con esta operación se retiran restos de tierra e impurezas que serán arrastradas por el flujo de agua hasta un tanque colector.

- Pelado y clasificado.- Esta operación se realizará mediante abrasión (método mecánico), ya que es el más apropiado para los tubérculos de cáscara delgada. Consiste en someter el material a rozamiento con rodillos, operación que, mediante la fricción, les irá arrancando la cáscara, para luego eliminarla por corrientes de agua. La velocidad de retención del material dentro de la máquina se encuentra 
regulada por un tornillo interno, controlado por un motor de velocidad graduable.

- Cortado.- Se utilizará una máquina rebanadora para poder obtener rodajas de forma y tamaño uniforme, de aproximadamente 2 milímetros de espesor. El corte se hará de manera transversal al tubérculo. La máquina elegida permite diversos tipos de corte, con ruedas cortadoras (cuchillas) intercambiables, para rebanadas lisas, onduladas y para corte "juliana".

- Fritura.- Es la operación central del proceso. La fritura será la conocida como deep frying, ya que las rodajas de yacón serán sumergidas dentro del aceite de palma. El tipo de freidora elegida consiste en un túnel por el cual las rodajas pasarán sobre una faja transportadora sumergida en aceite de fritura, cuya velocidad es regulable de acuerdo con los requerimientos.

- Escurrido.- Las hojuelas serán trasladadas en una faja transportadora que tendrá pequeños orificios, los cuales permitirán tanto que se escurra el aceite más rápidamente como su enfriamiento.

- Embolsado y sellado.- Las hojuelas serán embolsadas en empaques de 50 gramos. Las bolsas llevarán la siguiente información:

- Nombre y marca del producto.

- Información nutricional e ingredientes y peso neto.

- Fecha de elaboración y caducidad.

- Información de contacto de la empresa fabricante.

- Registro sanitario y otros.

Finalmente, las bolsas serán selladas herméticamente. Ambas operaciones se llevan a cabo en una sola máquina, compuesta por cabezales dosificadores para el embolsado y barras selladoras en caliente, que maximizan la transferencia de calor y reducen las desviaciones de temperatura, para de esta manera obtener un sellado adecuado.

\subsection{Proceso de producción}

En este inciso se presentan el Diagrama de Operaciones de Proceso y el Diagrama de Flujo. 


\section{Diagrama 1}

Diagrama de Operaciones de Proceso: Fabricación de snacks de yacón

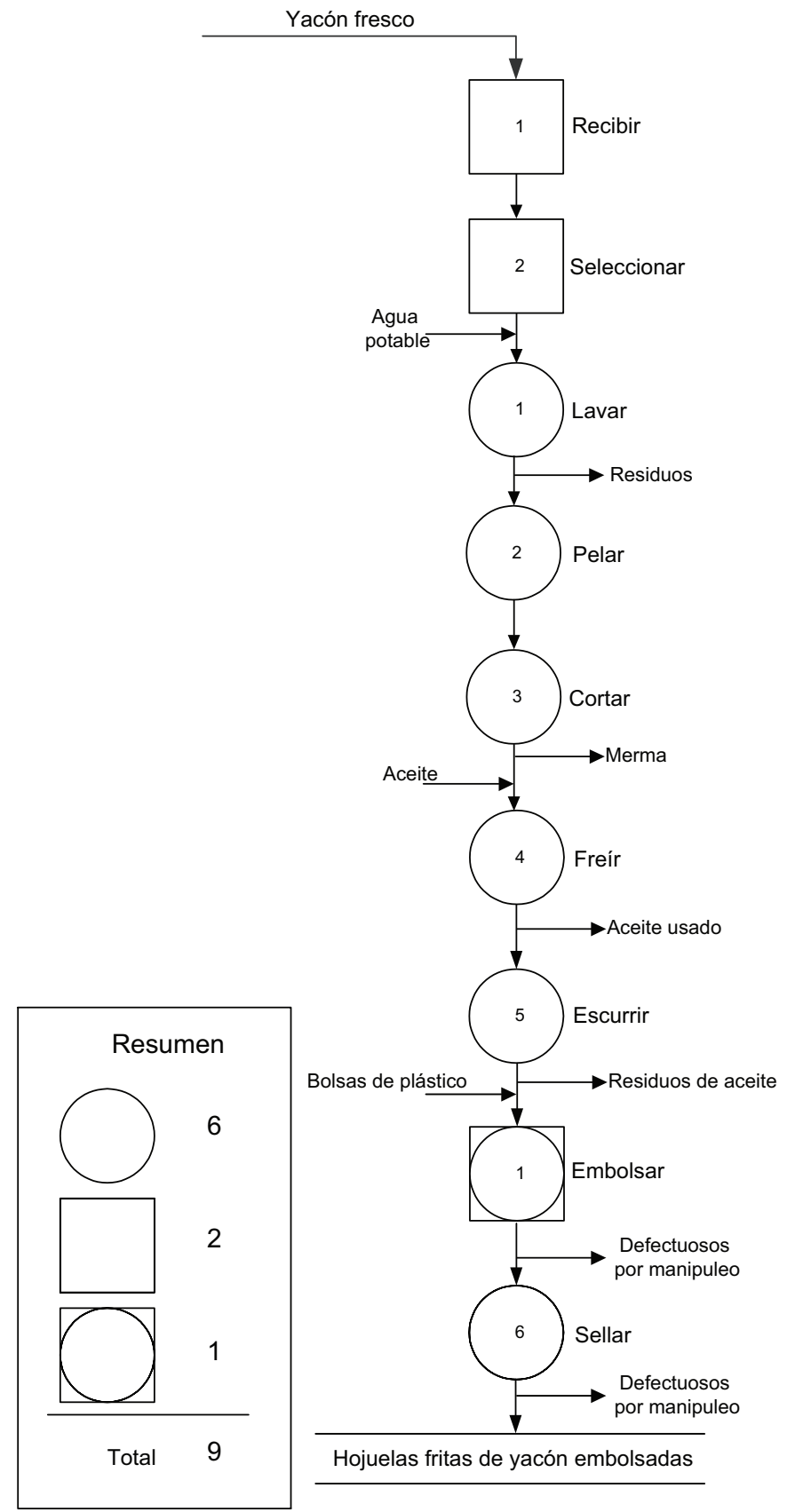

Elaboración propia. 
Estudio para la instalación de una fábrica de snacks a partir de la fritura de yacón

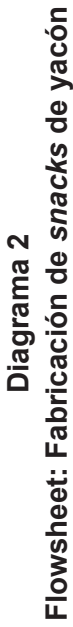

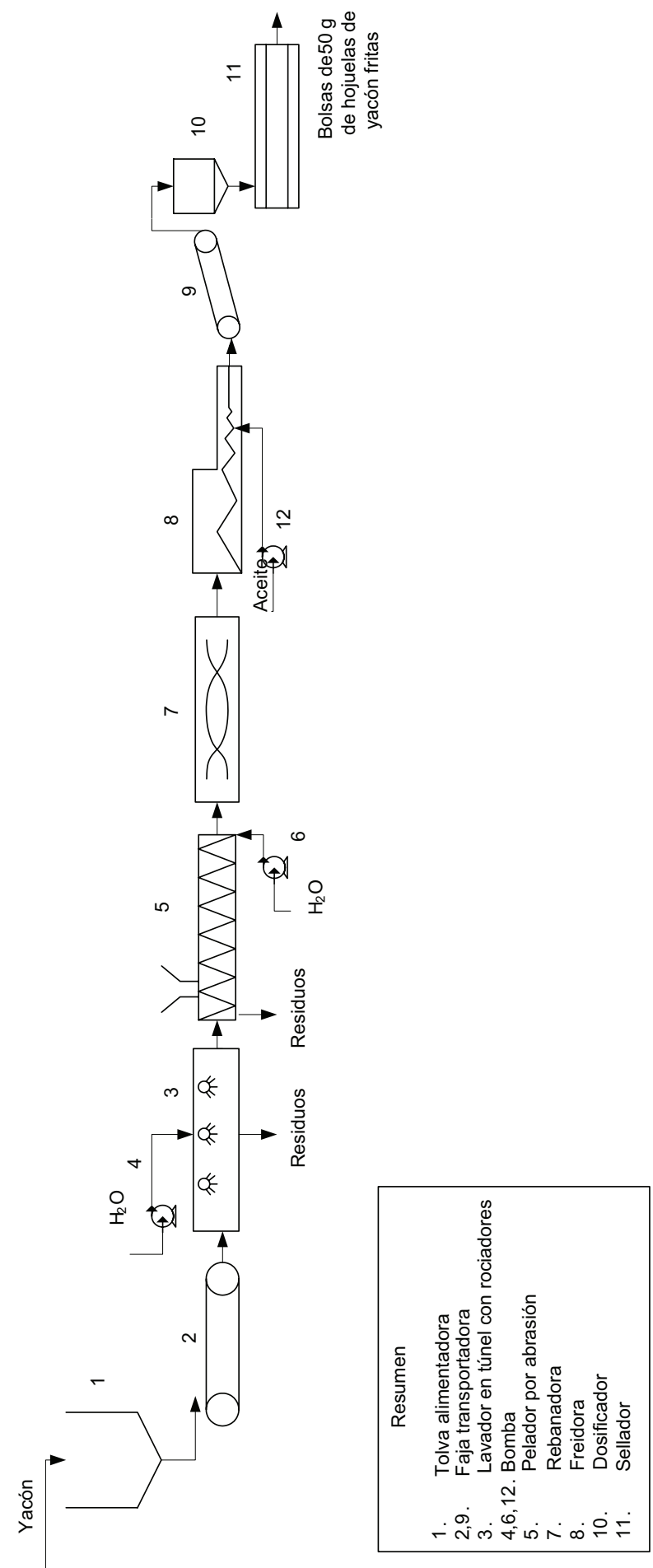

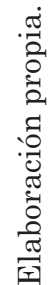




\section{INVERSIONES Y ASPECTOS ECONÓMICO-FINANCIEROS}

Tabla 15

Inversión total

\begin{tabular}{lrc}
\hline & Dólares & $\%$ \\
\hline Activos fijos tangibles & 1.016 .658 & 80 \\
Activos fijos intangibles & 20.178 & 2 \\
Capital de trabajo & 234.303 & 18 \\
Inversión total & 1.271 .139 & 100 \\
\hline
\end{tabular}

Fuente: Datos del proyecto. Elaboración propia.

La estimación de costos incluye los siguientes ítems:

Tabla 16

Estimación de costos

\begin{tabular}{|c|c|}
\hline Activos fijos tangibles & Activos fijos intangibles \\
\hline $\begin{array}{l}\text { - Estudio de factibilidad } \\
\text { - Constitución y organización } \\
\text { - Reclutamiento y capacitación de personal } \\
\text { - Gastos financieros } \\
\text { - Gastos administrativos } \\
\text { - Gastos de puesta en marcha }\end{array}$ & $\begin{array}{l}\text { - Terreno } \\
\text { - Infraestructura } \\
\text { - Maquinaria y equipos } \\
\text { - Muebles y equipos de oficina }\end{array}$ \\
\hline \multicolumn{2}{|c|}{ Capital de trabajo } \\
\hline \multicolumn{2}{|c|}{ Gasto total anual $x$ días ciclo } \\
\hline \multicolumn{2}{|l|}{365} \\
\hline
\end{tabular}

Elaboración propia.

La inversión total será financiada con fondos de la Corporación Financiera de Desarrollo (Cofide), hasta el tope de 60\%, con lo cual el monto total quedaría dividido de la siguiente manera:

$$
\text { US } \$ 1.271 .139=\mathrm{US} \$ 762,683(60 \%)+\mathrm{US} \$ 508,455(40 \%)
$$

El 60\% de la inversión total será financiada mediante el Programa Multisectorial de Inversión: Probid, con el cual Cofide, al funcionar como banco de segundo piso, otorga un redescuento a la institución 
financiera intermediaria. Para ello, se eligió al Banco de Crédito del Perú como institución financiera intermediaria. Las tasas de interés anual son las siguientes:

Tasa BCP-subprestamista: Prime Rate NY $+8,75 \%=12 \%$

Tasa Cofide-BCP: Libor $^{8}+2,25 \%=3,33 \%$

La tasa de interés a pagar finalmente es de 15,33\% anual. Asumiendo un costo de capital (Kp) del 17\%, se calcula el costo promedio ponderado de capital (CPPC):

Tabla 17

Costo promedio ponderado de capital

\begin{tabular}{lccc}
\hline \multicolumn{1}{c}{ CPPC } & Proporción & Tasa & Porcentaje \\
\hline Aporte & 40 & 17,00 & 6,80 \\
Financiamiento & 60 & 15,33 & 9,20 \\
\hline & & CPPC & 16,00 \\
\cline { 3 - 4 }
\end{tabular}

Nota: Para efectos académicos y de practicidad, no se considera el escudo fiscal.

Elaboración propia.

\section{EVALUACIÓN ECONÓMICO-FINANCIERA}

Se plantearon tres escenarios de evaluación, considerando el 90\% de ventas en el Optimista, 70\% en el Probable y 50\% en el Pesimista. A continuación se presentan los principales indicadores económicos (tasa $=17 \%)$ y financieros $($ tasa $=16 \%)$ :

Tabla 18

Indicadores económicos

\begin{tabular}{cccc}
\hline & Optimista & Probable & Pesimista \\
\hline VAN & S/. 11.448 .572 & S/. 4.116 .047 & S/. -3,441,616 \\
TIR & $69 \%$ & $36 \%$ & $\mathbf{- 2} \%$ \\
B/C & 4,75 & 2,46 & $\mathbf{0 , 0 9}$ \\
PR & 3 & 5 & $\mathbf{1 0 +}$ \\
\hline
\end{tabular}

Elaboración propia.

7 3,25\%. Tomado de: http://www.wsjprimerate.us/

8 1,08\%. Tomado de: http://www.bankrate.com/rates/interest-rates/libor.aspx 
Tabla 19

Indicadores financieros

\begin{tabular}{lccc}
\hline & Optimista & Probable & Pesimista \\
\hline VAN & S/. 12.135 .964 & S/. 4.501 .174 & S/. 4.501174 \\
TIR & $69 \%$ & $36 \%$ & $\mathbf{- 2 \%}$ \\
B/C & 4,93 & 2,56 & $\mathbf{0 , 1 1}$ \\
PR & 3 & 5 & $\mathbf{1 0 +}$ \\
\hline
\end{tabular}

Elaboración propia.

Se puede concluir que el proyecto propuesto representa una oportunidad, al mostrarse rentable en los escenarios optimista y probable evaluados, teniendo ambos un enfoque conservador al considerar ventas por debajo de lo planificado.

\section{EVALUACIÓN SOCIOECONÓMICA}

El proyecto tendrá un impacto positivo en el entorno social al generar empleo bien remunerado con utilidades, además de permitir el desarrollo y explotación de un tubérculo subestimado por la generación actual. Generará trabajo y desarrollo para todos los actores de la cadena, principalmente para las comunidades productoras que verán un incremento importante de la demanda y sobre las cuales se priorizará la inversión y el trabajo conjunto.

\section{CONCLUSIONES Y RECOMENDACIONES}

- El proyecto es aparentemente viable, después de haber analizado tres escenarios posibles con distintos tipos de rendimiento.

- En el desarrollo del estudio se tuvieron limitaciones en cuanto a información. Dentro de este marco, se trabajó sobre información real y actualizada, tomando en varios casos el procesamiento de hojuelas fritas de papa como punto de referencia al ser este proceso bastante similar al propuesto.

- Después de realizar el estudio de mercado en la ciudad de Lima, se concluye que existe un nicho dispuesto a consumir el producto propuesto. Sin embargo, se recomienda una mayor profundización de dicho estudio a fin de tener información más representativa y con el menor sesgo posible. 
- La materia prima elegida presenta numerosas ventajas, como su alto rendimiento y sus considerables atributos beneficiosos para la salud, que al ser aprovechadas adecuadamente pueden convertirse en alternativas de desarrollo de las comunidades menos favorecidas del país. En todo caso, se deben realizar mejores pruebas de laboratorio a fin de corroborar la viabilidad técnica.

- A pesar de haber conseguido varias cotizaciones y estimados, es necesario contar con valores más exactos de precios y demás costos, para poder efectuar un óptimo análisis económico y financiero.

- A fin de lograr mayor desarrollo del producto, será una interesante opción elaborar diferentes presentaciones del producto, además de combinarlo con otras variedades de materia prima a fin de obtener productos finales con mayor valor nutricional.

\section{REFERENCIAS}

1. Armas, Granados \& Puente (2004). Snacks de nueces de Brasil. Lima: Universidad ESAN.

2. Banco de Crédito del Perú (2010). Financiación, empresas, mediano plazo. Recuperado el 17 de octubre de 2011, de http://www.viabcp.com/zona_publica/03_empresa/interna. asp?SEC=3\&JER=777\&ENL=1001

3. Corporación Financiera de Desarrollo (2006). Tasas de interés y comisiones a las instituciones financieras intermediarias de los programas y líneas de financiamiento Cofide. Recuperado el 23 de octubre de 2011, de http://www.cofide.com.pe/textos/ tasas_18042006.pdf

4. Doig C., Gabriela (2011). Estudio de prefactibilidad para la instalación de una planta fabricadora de snacks a partir de fritura de yacón. Tesis para optar el título de ingeniera industrial. Lima: Universidad de Lima.

5. EsSalud (2005). Protección social en el sector agrario peruano: Perspectivas y estrategias. Recuperado el 10 de octubre de 2011, de http://white.oit.org.pe/spanish/260ameri/oitreg/activid/proyectos/actrav/proyectos/pdf/sectoragrario_2005.pdf 
6. Grau, A. \& Aragón, M. (2000, junio). Especies exóticas como recurso para las aves en bosques secundarios de las yungas. Árboles Exóticos de las Yungas Argentinas, 12, 21-35.

7. Hill, Charles W. L. (2005). Administración estratégica. México, D. F.: McGraw-Hill.

8. Instituto Nacional de Estadística e Informática (2009). Perú: Anuario de estadísticas ambientales. Recuperado el 24 setiembre de 2011, de http://www1.inei.gob.pe/biblioineipub/bancopub/Est/ Lib0800/Libro.pdf

9. Manrique, I. \& Hermann, M. (2003). El potencial del yacón en la salud y la nutrición. Cochabamba, XI Congreso Internacional de Cultivos Andinos. 15-19 de octubre.

10. Manrique, I., Párraga, A. \& Hermann, M. (2005). Yacon syrup: principles and processing. Recuperado el 24 de setiembre de 2011, de http://www.cipotato.org/artc/cip_crops/1919-Yacon_Syrup.pdf

11. Manrique, I. \& Rivera, D. (2005). Zumo de yacón: ficha técnica. Recuperado el 24 de septiembre de 2011, de http://www.cipotato. org/artc/cip_crops/docs/fichazumoyacon.pdf

12. Ministerio de la Producción. Crecemype. Recuperado el 26 de setiembre de 2011, de http://www.crecemype.pe

13. Ministerio de la Producción. Boletín de Estadistica Industrial Mensual. Recuperado el 26 de setiembre de 2011, de http://www. produce.gob.pe

14. PNUD (2006). La democracia en el Perú: El mensaje de las cifras. Recuperado el 24 de setiembre de 2011, de http://www. proinversion.gob.pe/Default.aspx?ARE $=0 \& \mathrm{PFL}=0$

15. Portal Agrario Ancash (2008). Recuperado el 3 de agosto del 2009, de http://www.agroancash.gob.pe/public/articulos/aip2008/temas /variedades.htm

16. Seminario, J. (1995). Descriptores básicos para la caracterización del chago (Mirabilis expansa), el yacón (Smallanthus sonchifolius) y la achira (Canna edulius). I Congreso Peruano de Cultivos Andinos. Huamanga, Ayacucho.

17. Seminario, J., Valderrama, M., \& Manrique, I. (2003). El yacón: fundamentos para el aprovechamiento de un recurso promisorio. 
Lima: Centro Internacional de la Papa / Universidad Nacional de Cajamarca / Agencia Suiza para el Desarrollo y la Cooperación.

18. Superintendencia Nacional de Aduanas y Administración Tributaria. Portal Aduanet. Recuperado el 26 de setiembre de 2011, de http://www.sunat.gob.pe

19. Universidad Nacional Agraria La Molina (2010). Programa de Investigación y Proyección Social en Raíces y Tuberosas: Yacón. Recuperado el 25 de setiembre de 2011, de http://www.lamolina. edu.pe/Investigacion/programa/yacon/Yacon.htm

20. Valderrama, M., Acero, A., \& Díaz, A. (2006). Experiencia de técnicas de propagación vegetativa para la producción comercial de yacón. Recuperado el 25 de setiembre de 2011, de http://www.cosude. org.pe/es/Pagina_principal/ressources/resource_es_97928.pdf 